\title{
A Search Patterns Switching Algorithm for Block Motion Estimation
}

\author{
Ka-Ho Ng, Lai-Man Po, Ka-Man Wong, Chi-Wang Ting, and Kwok-Wai Cheung
}

\begin{abstract}
Center-biased fast motion estimation algorithms, e.g., block-based gradient descent search and diamond search, can perform much better than coarse-to-fine search algorithms, such as 2-D logarithmic search and three-step search. The latter type of algorithms, however, is more suitable for handling large motion content. To combine the advantages of both types of algorithms, an adaptive algorithm performing search patterns switching (SPS) is proposed in this paper. The proposed SPS algorithm classifies the motion content of a block using a simple yet efficient motion content classifier called error descent rate. Unlike other classifiers with heavy overhead, this classifier requires only the searching of a few points in the search window and then a division operation. Experimental results show that the proposed SPS algorithm is very robust.
\end{abstract}

Index Terms-Block matching, motion estimation, video coding.

\section{INTRODUCTION}

Block matching algorithms (BMAs) have been widely used in motion estimation (ME) for various video coding standards such as the H.26X series [1]. Full search (FS) can obtain the optimal motion vectors (MVs) but it is very slow. Many fast BMAs (FBMAs) have been proposed to speed up the ME process by reducing the number of search points based on two approaches. One approach, as employed in 2-D logarithmic search (2-DLOG) [2] and three-step search (3SS) [3], uses coarse-to-fine searching to reduce the number of search points. This approach is efficient for large-motion video sequences because in these sequences the search points are evenly distributed over the search window and thus the global minima far away from window centers can be located more efficiently. For small motions, this approach is less efficient.

The second approach utilizes the center-biased characteristic of MVs. According to an analysis on motion vector distribution in [4], about half of the macro-blocks are stationary and most of the MVs lie within the central $5 \times 5$ region of the search window. Algorithms such as new three-step search (N3SS) [5], four-step search (4SS) [6], block-based gradient descent search (BBGDS) [7], diamond search (DS) [8], and cross diamond search (CDS) [4] use center-biased

Manuscript received January 26, 2008; revised June 6, 2008. First version published March 16, 2009; current version published June 10, 2009. This work was supported by a grant from City University of Hong Kong, Hong Kong SAR, China under Project 9041251 (CityU 119207). This paper was recommended by Associate Editor L. Chen.

K.-H. Ng, L.-M. Po, K.-M. Wong, and C.-W. Ting are with the Department of Electronic Engineering, City University of Hong Kong, Hong Kong SAR, China (e-mail: kahomike@gmail.com; eelmpo@cityu.edu.hk; kmwong@ee.cityu.edu.hk; cwting@cityu.edu.hk).

K.-W. Cheung is with the Department of Computer Science, Chu Hai College of Higher Education, Hong Kong SAR, China (e-mail: kwcheung@chuhai.edu.hk).

Digital Object Identifier 10.1109/TCSVT.2009.2017414 search patterns to utilize the center-biased global minima distribution. Compared with coarse-to-fine search algorithms, a substantial reduction of search points can be achieved for small motion sequences. For videos with large motions, however, they are subject to quality degradation because they can be easily trapped in local minima.

Adaptive algorithms combine the advantages of the above two approaches by using different search patterns according to the motion content of a block. The performance of an adaptive algorithm depends on the accuracy of its motion content classification.

In this paper, an adaptive search patterns switching (SPS) algorithm is proposed. A simple yet efficient motion content classifier based on error descent rate (EDR) is developed for the search patterns switching decision.

The rest of the paper is organized as follows. Section II gives an overview on the various motion content classifiers. EDR is discussed in Section III. The proposed SPS algorithm based on EDR is described in Section IV. Experimental results are given in Section V, while Section VI gives the conclusions.

\section{Motion Content Classifier}

An adaptive ME algorithm can select between search patterns or search strategies for different motion contents. The motion content of a block can be classified by a motion content classifier. Various types of motion content classification have been proposed.

\section{A. Zero Motion Content Classifier}

Zero motion content blocks, i.e., the blocks with zero motion vectors (ZMVs), exist in most video sequences [4]. If a block is classified as a zero motion block, subsequent motion search can be skipped. Some early termination algorithms compare the distortion of a block at the zero motion point with a threshold. The threshold value can be predefined based on statistical results [9] or determined dynamically during the encoding process [10]. The main challenge of a zero motion content classifier is to find a universal threshold value for different video sequences.

\section{B. Geometric Motion Content Classifier}

The N3SS uses eight more search points in its initial search compared with 3SS. The initial search pattern can be considered as the geometric motion content classifier of $\mathrm{N} 3 \mathrm{SS}$. If the minimum distortion position is near the window center, the block is classified as a small motion block and a very compact search pattern similar to that of BBGDS is 
used for the subsequent search. Otherwise, N3SS employs the coarse-to-fine search used in 3SS to search the large MVs.

Similar to N3SS, the efficient three-step search (E3SS) [11] uses its initial search pattern as the motion content classifier. Either small diamond search (SDS) or 3SS will be selected for subsequent search according to the motion content classification. Adaptive double-layered initial search pattern algorithm [12] also uses the initial search pattern as the classifier. Geometric motion classifier uses a large number of initial search points. Although the position of the global minimum can be estimated more accurately, the speed is slow, particularly in small motion sequences.

\section{Motion Content Classifier Using MV Prediction}

Motion content classifiers may use the information of spatial or temporal neighbor MVs to determine the starting search position and the search pattern [13], [14]. For example, the algorithm proposed in [14] classifies a block into three categories. Either FS, 3SS, or 4SS will then be selected. In these algorithms, the motion content classification using predicted MV information can remove the temporal and spatial redundancy between blocks. However, motion content classification using MV prediction requires much more data storage and computational overhead.

A-TDB algorithm proposed in [15] uses another motion content classifier called predicted profit list, which is a sorted list of the search center distortions of all the blocks in a frame. Experimental results show that the predicted profit list is robust. However, the storage and sorting overhead of the list increases the complexity of the classification process.

\section{ERROR DESCENT RATE}

By the unimodal error surface assumption, block distortion monotonically decreases towards the global minimum. It can be further assumed that a global minimum point has a greater effect on its nearby error surface than the error surface further away from it. This effect is reflected by the rate of decrease in distortion. The nearer the global minimum, the faster the decrease in distortion. Fig. 1 shows this effect. The rate of decrease in distortion can be quantified by the slope of the distortion surface. Using the same assumption, the position of a global minimum in an ME search window affects the decrease in distortion at the search window center. Fig. 2 shows four hypothetical positions of a global minimum. The decrease in distortion near the search window center is represented by the slope of the curve within the distance $d$. For the global minima at position 1 to 4 , the slopes of the curves are approximated by $(A-B) / d,(B-C) / d,(C-D) / d$, and $(D-E) / d$ respectively. An important feature of this assumption is that the distance of the global minimum from the search window center can be estimated by comparing the slopes. This is the basis of the proposed motion content classifier.

The error descent slopes at the center of the search window can be approximated by the following method. First, find

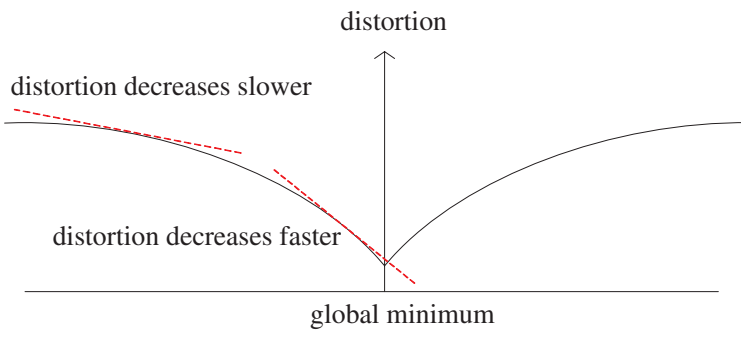

Fig. 1. Rate of decrease in distortion near a global minimum point.

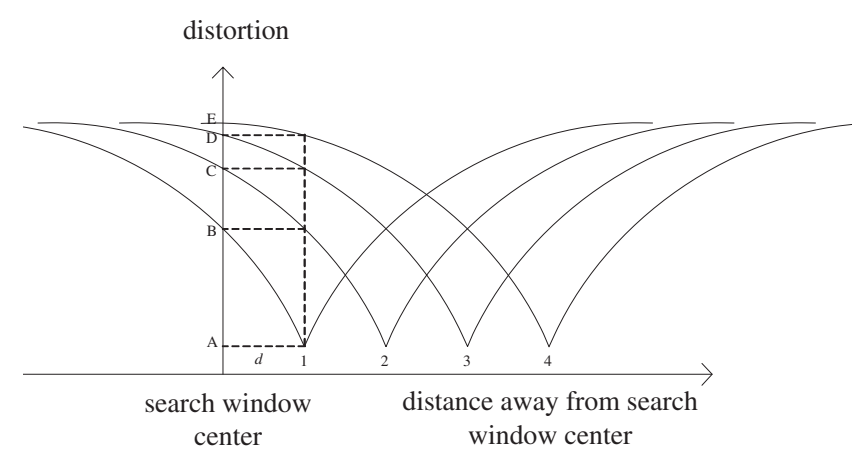

Fig. 2. Effects of the position of a global minimum on the slope of error descent around the search window center.

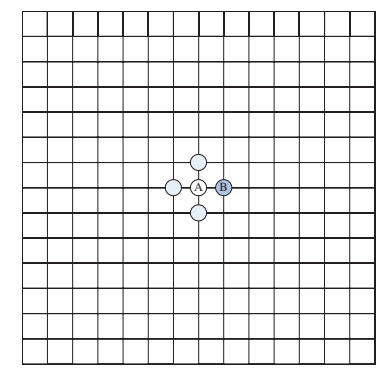

Fig. 3. Distortion difference between the center point and its minimum distortion neighbor.

the distortion of the search window center point $\mathrm{A}$ and note it as $D_{A}$. Then calculate the distortions of its four adjacent points. Among the four points, the one with the minimum distortion is recognized as point $\mathrm{B}$ and its distortion is noted as $D_{B}$. This is shown in Fig. 3. The slope of error descent is then calculated as $\left(D_{B}-D_{A}\right)$, as all of the four adjacent points are one pixel away from the center point. By the above method, the steepest error descent slope is used to estimate whether the global minimum is near or far away from the search window center. From plots of the average values of $\left(D_{B}-D_{A}\right)$ against the Euclidean distances between the global minima and the search window centers, we can observe an increasing trend of $\left(D_{B}-D_{A}\right)$ with increasing distance between global minima and search window centers. However, it is not easy to use the values $\left(D_{B}-D_{A}\right)$ because they are very much dependent on the video content.

Although the difference between $D_{B}$ and $D_{A}$ is video content dependent, the EDR, defined as the ratio between $D_{B}$ and $D_{A}$, is relatively independent of the video content. The 


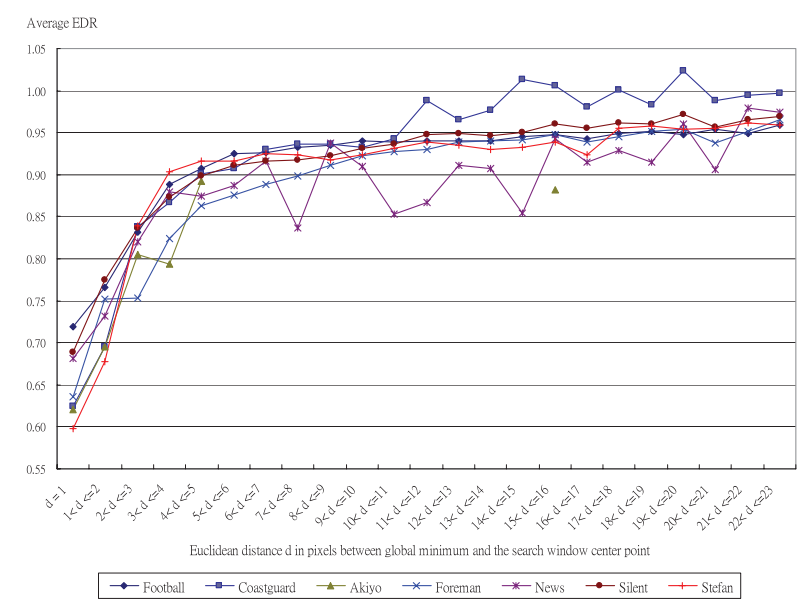

Fig. 4. Average EDRs versus distances between global minima and search window centers for CIF sequences.

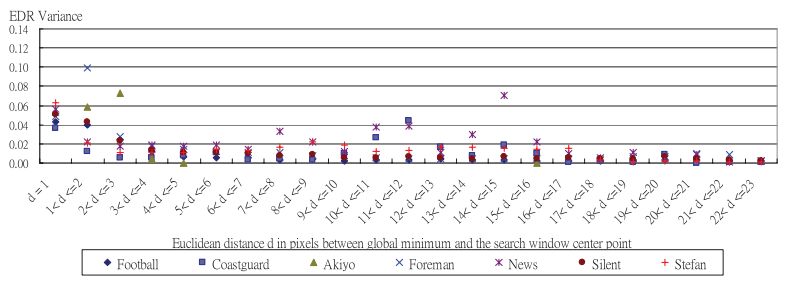

Fig. 5. EDR variances versus distances between global minima and search window centers for CIF sequences.

EDR is defined as

$$
\mathrm{EDR}=\mathrm{D}_{\mathrm{B}} / \mathrm{D}_{\mathrm{A}}
$$

where $D_{B}$ is the minimum distortion of the four adjacent points of the search window center and $D_{A}$ is the distortion at the search window center. For the case $D_{B}=60$ and $D_{A}=100$, the error descent rate is 0.6 or the distortion at point $\mathrm{B}$ is $40 \%$ less than the distortion at search window center point A. EDR values of 0.8 and 0.6 refer to $20 \%$ and $40 \%$ drop in distortion, respectively. As a ratio between the distortion values at different positions on an error surface, EDR can be considered as a normalized slope of error descent and is independent of different motion contents as shown from Figs. 4-7.

Figs. 4 and 6 plot the average values of EDR against the Euclidean distances between the global minima and the search window centers for several CIF, QCIF, and CCIR601 test video sequences. Details of the test sequences used are given in Table III. Figs. 5 and 7 show the EDR variances for the sequences. The average EDRs for $d=0$ are much larger than one and are not shown in the figures. EDR being larger than one (i.e., $D_{B}>D_{A}$ ) can mean that the center point is in fact a local minimum. Based on the center-biased characteristic of MVs, it is safe to assume that this local minimum is also the global minimum. As shown in Figs. 4 and 6, there is a general trend of increasing EDR value with increasing distance between global minima and search window centers. This shows that EDR is a good indicator of the proximity of the global minimum to the search window center and also provides strong support to the assumption used in Figs. 1 and 2. The variances of EDR shown in Figs. 5 and 7 are

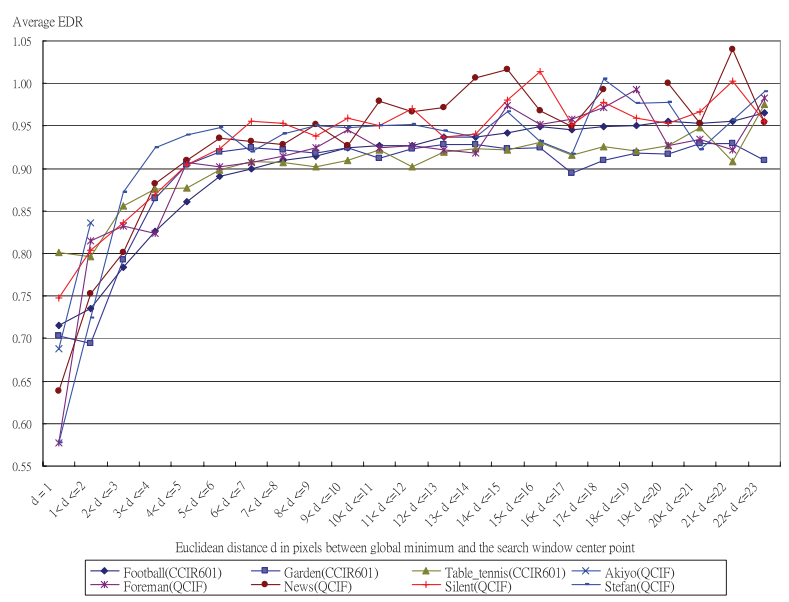

Fig. 6. Average EDRs versus distances between global minima and search window centers for QCIF and CCIR601 sequences.

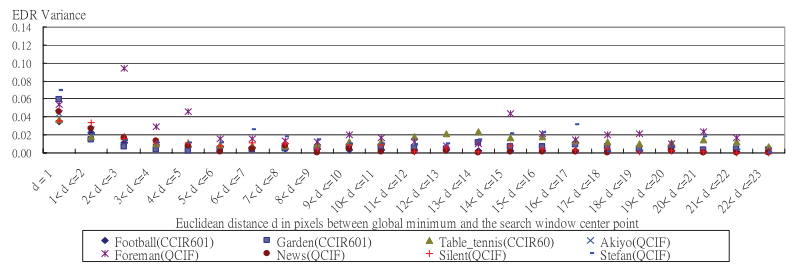

Fig. 7. EDR variances versus distances between global minima and search window centers for QCIF and CCIR601 sequences.

generally low and video content independent. All the test video sequences, which include small, medium, and large motion contents, show the same trend of increasing EDR values with increasing MV lengths. Thus, EDR can be used reliably to estimate the proximity of a global minimum.

Most of the sequences in Figs. 4 and 6 have global minima within a distance of three pixels from the center if their EDR is below 0.85 . In other words, if the distortion value has more than $15 \%$ decrease at the center of the search window, the global minimum is usually near the search window center. For global minima with a distance of more than 10 pixels from the center, they usually have $1>$ EDR $>0.9$, which means less than $10 \%$ error descent at the center. This further proves that EDR is a good estimator of the distance between the global minimum and the search window center.

\section{Search Pattern Switching}

The SPS algorithm using EDR as motion content classifier is shown in Fig. 8. This algorithm uses the EDR at the center of the search window to estimate whether the global minimum is near the center. If so, a search pattern suitable for small motion searching will be used. Otherwise, a search pattern suitable for searching large motions will be applied.

As shown in Fig. 8, EDR at the search window center is first evaluated. If EDR $>1$, all the four immediately adjacent points have distortion higher than the search window center point. According to the center-biased property of MVs [4], a high percentage of MVs are within one pixel distance from the search window center. Thus, if EDR $>1$, zero motion vector ( $\mathrm{ZMV})$ case is assumed, and the search stops for this 


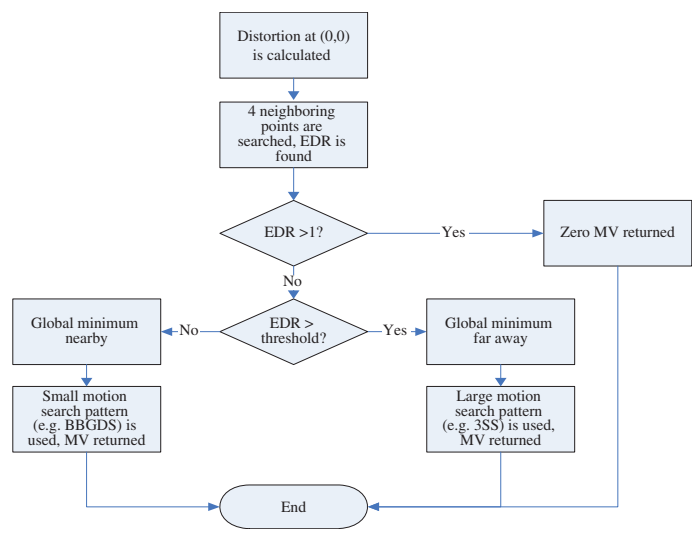

Fig. 8. Flow diagram of the search patterns switching (SPS) algorithm.

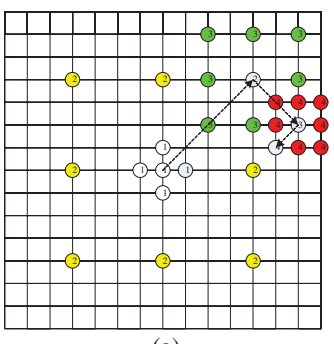

(a)

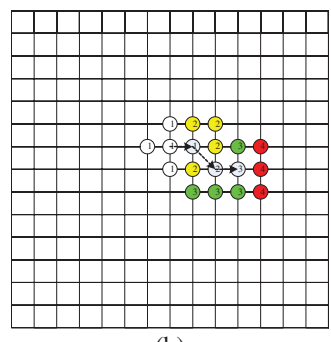

(b)
Fig. 9. Example of (a) $3 S S$ if EDR $>$ T. (b) BBGDS if EDR $\leq \mathrm{T}$.

block. Given that EDR $\leq 1$, if EDR is larger than a threshold $\mathrm{T}$ (say 0.9 ), the global minimum is more likely to be at the outer area of the search window. 3SS will then be used for motion search. If EDR $\leq \mathrm{T}$, the global minimum is more likely to be close to the center. Block-based gradient descent search (BBGDS) will then be selected instead. Fig. 9(a) and (b) show examples of subsequent search using 3SS and BBGDS for locating the global minimum at the outer area and near the center, respectively.

SPS algorithm can employ other search patterns. Typical combinations include using DS instead of BBGDS for small motion search and using 4SS instead of 3SS for large motion search. SPS combinations can be represented as SPS(X, Y), where $\mathrm{X}$ and $\mathrm{Y}$ are the names of the small and large search patterns, respectively. Figs. 10 and 11 show examples of small motion search using DS for SPS(DS, Y) and large motion search using 4SS for SPS(X, 4SS), respectively. Various combinations for SPS algorithm are evaluated. Basically, the best combinations are those using center-biased search patterns, e.g., BBGDS and DS, for small motion search and coarse-tofine search patterns, e.g., 3SS and 4SS, for large motion search. For each SPS combination, an optimum threshold value $\mathrm{T}$ can be found by empirical method which will be discussed in Section V. It is found that the optimum threshold values for all tested combinations are in the range of 0.85-0.95. This result matches with the assumption and statistics in Section III.

The SPS algorithm notation can be rewritten as SPS(X, Y, $\mathrm{T}$ ), where $\mathrm{T}$ is the threshold. The accuracy of SPS algorithm in motion content classification is given in Table I and II.

Table I gives the distributions of MVs found by FS and that estimated by EDR-based SPS. The second column contains the percentages of ZMVs found by FS. The third column contains

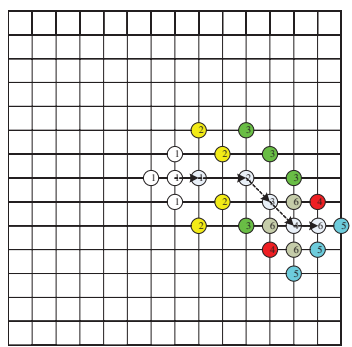

Fig. 10. Example of DS if EDR $\leq \mathrm{T}$, for SPS (DS, Y).

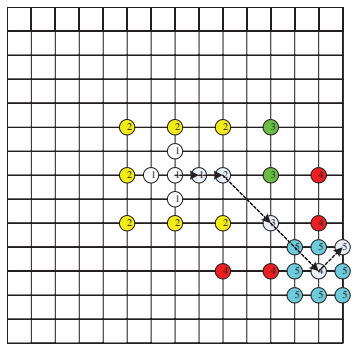

Fig. 11. Example of $4 \mathrm{SS}$ if EDR $>\mathrm{T}$, for SPS $(\mathrm{X}, 4 \mathrm{SS})$.

the percentages of MVs with lengths smaller than or equal to five pixels. The fourth column contains the percentages of MVs with lengths larger than five pixels. The fifth column contains the percentages of ZMVs determined by the condition EDR $>1$. The sixth and seventh columns, respectively, contain the percentages of small and large MVs, estimated by SPS. The threshold value used is 0.9 .

The results show that the distribution estimated by SPS is fairly close to that found by FS. Table II shows the classification accuracy of SPS. The result of SPS estimation is compared with that of the FS. The percentages of correct estimations are tabulated. The estimation accuracy ranges from $76.03 \%$ to $98.80 \%$. The estimation accuracy is lower for complex motion sequences, e.g., Stefan. Nevertheless, considering the simplicity, EDR is still a very efficient motion content classifier for SPS algorithm.

\section{Performance Evaluation}

Experiments were conducted to find the optimum threshold values for various SPS combinations. The performance of SPS is also compared with that of other algorithms. The details of the sequences used are listed in Table III. The block size is $16 \times 16$ pixels. The frame structure is IPPP and the search range is \pm 16 pixels. Simulation results are expressed in average number of search points used per block and average PSNR (dB) per frame.

\section{A. Optimum Threshold for SPS Combinations}

Table IV lists the average PSNR per frame and the average number of search points used per block for the combination SPS(BBGDS, 3SS, T) using different threshold values $\mathrm{T}$ for the CIF sequences. Searches will not be performed outside the frame. In other words, blocks on the frame boundary have clipped search windows. Therefore, the minimum number of search points used by SPS is three for corner blocks and four for boundary blocks, during ZMV returns. 
TABLE I

COMPARISON OF MV DISTRIBUTIONS FOUND BY FS AND BY EDR

\begin{tabular}{c|c|c|c|c|c|c}
\hline & \multicolumn{3}{|c|}{ Full search } & \multicolumn{3}{c}{ Estimation Using EDR } \\
\hline & $\%$ of ZMV & $\%$ of MVs <= 5 & $\%$ of MVs > 5 & $\%$ of ZMV & $\%$ of small MVs & $\%$ of large MVs \\
\hline Football & $30.21 \%$ & $22.13 \%$ & $47.66 \%$ & $33.42 \%$ & $23.47 \%$ & $43.11 \%$ \\
\hline Coastguard & $10.05 \%$ & $86.92 \%$ & $3.04 \%$ & $10.55 \%$ & $81.60 \%$ & $7.85 \%$ \\
\hline Akiyo & $92.86 \%$ & $7.14 \%$ & - & $93.02 \%$ & $5.94 \%$ & $1.03 \%$ \\
\hline Foreman & $21.43 \%$ & $59.43 \%$ & $19.14 \%$ & $25.23 \%$ & $55.81 \%$ & $18.96 \%$ \\
\hline News & $81.50 \%$ & $16.86 \%$ & $1.64 \%$ & $82.32 \%$ & $13.35 \%$ & $4.33 \%$ \\
\hline Silent & $82.85 \%$ & $13.14 \%$ & $4.01 \%$ & $84.29 \%$ & $10.45 \%$ & $5.26 \%$ \\
\hline Stefan & $29.32 \%$ & $46.75 \%$ & $23.93 \%$ & $34.78 \%$ & $36.61 \%$ & $28.61 \%$ \\
\hline Mobile & $27.03 \%$ & $70.75 \%$ & $2.22 \%$ & $28.78 \%$ & $65.13 \%$ & $6.09 \%$ \\
\hline
\end{tabular}

TABLE II

Motion Content Classification Accuracy of EDR (Threshold T = 0.9)

\begin{tabular}{c|c|c|c|c|c|c|c|c}
\hline & Football & Coastguard & Akiyo & Foreman & News & Silent & Stefan & Mobile \\
\hline $\begin{array}{c}\text { correct } \\
\text { estimation } \\
(\%)\end{array}$ & $81.17 \%$ & $93.20 \%$ & $98.80 \%$ & $82.29 \%$ & $95.11 \%$ & $94.19 \%$ & $76.03 \%$ & $91.78 \%$ \\
\hline
\end{tabular}

TABLE III

VIDEO SEQUenCES USED IN SimUlations

\begin{tabular}{c|c}
\hline Format & Sequences \\
\hline CIF $(352 \times 288,200$ frames $)$ & Akiyo, News, Coastguard, Foreman, Stefan, Football, Silent \\
\hline QCIF $(176 \times 144,300$ frames $)$ & Akiyo, Foreman, News, Silent, Stefan \\
\hline CCIR601 $(720 \times 486,100$ frames $)$ & Football, Garden, Table_tennis \\
\hline
\end{tabular}

TABLE IV

COMPARISON OF DIFFERENT T IN SPS (BBGDS, 3SS, T)

\begin{tabular}{c|c|c|c|c|c|c|c|c|c|c}
\hline & \multicolumn{2}{|c|}{ Akiyo } & \multicolumn{2}{c|}{ News } & \multicolumn{2}{c|}{ Coastguard } & \multicolumn{2}{c|}{ Foreman } & \multicolumn{2}{c}{ Stefan } \\
\hline $\begin{array}{c}\text { SPS } \\
(\text { BBGDS, 3SS, T })\end{array}$ & $\begin{array}{c}\text { PSNR } \\
(\mathrm{db})\end{array}$ & $\begin{array}{c}\text { \# of search } \\
\text { points }\end{array}$ & $\begin{array}{c}\text { PSNR } \\
(\mathrm{db})\end{array}$ & $\begin{array}{c}\text { \# of search } \\
\text { points }\end{array}$ & $\begin{array}{c}\text { PSNR } \\
(\mathrm{db})\end{array}$ & $\begin{array}{c}\text { \# of search } \\
\text { points }\end{array}$ & $\begin{array}{c}\text { PSNR } \\
(\mathrm{db})\end{array}$ & $\begin{array}{c}\text { \# of search } \\
\text { points }\end{array}$ & $\begin{array}{c}\text { PSNR } \\
(\mathrm{db})\end{array}$ & $\begin{array}{c}\text { \# of search } \\
\text { points }\end{array}$ \\
\hline $\mathrm{T}=0.70$ & 43.014 & 5.269 & 36.681 & 7.314 & 29.859 & 21.328 & 32.258 & 19.700 & 24.250 & 20.779 \\
\hline $\mathrm{T}=0.75$ & 43.015 & 5.219 & 36.693 & 7.063 & 29.860 & 18.583 & 32.270 & 18.634 & 24.251 & 20.186 \\
\hline $\mathrm{T}=0.80$ & 43.015 & 5.175 & 36.702 & 6.810 & 29.866 & 16.341 & 32.284 & 17.572 & 24.249 & 19.448 \\
\hline $\mathrm{T}=0.85$ & 43.018 & 5.126 & 36.706 & 6.567 & 29.877 & 14.410 & 32.295 & 16.547 & 24.247 & 18.459 \\
\hline $\mathrm{T}=0.90$ & 43.018 & 5.076 & 36.730 & 6.343 & 29.889 & 13.034 & 32.300 & 15.648 & 24.231 & 17.045 \\
\hline $\mathrm{T}=0.95$ & 43.020 & 5.029 & 36.694 & 6.108 & 29.864 & 12.248 & 32.243 & 14.895 & 24.036 & 15.119 \\
\hline $\mathrm{T}=0.975$ & 43.022 & 5.002 & 36.673 & 5.980 & 29.837 & 11.983 & 32.138 & 14.621 & 23.770 & 14.025 \\
\hline
\end{tabular}

Table IV shows that the number of search points used is decreasing with increasing threshold values. More blocks will be classified as small motion blocks with higher threshold value. BBGDS, which is more easily trapped in a local minimum near the search center, will be used for these blocks. Therefore, although fewer search points are used, the quality degradation is severe. With a lower threshold value, more search points are needed because the large motion search 3SS converges slowly. It can be observed that $\mathrm{T}=0.9$ is an optimum threshold for SPS(BBGDS, 3SS, T). For the sequences Football, Coastguard, Foreman, and News, T $=0.9$ achieves the highest PSNR. With $\mathrm{T}>0.9$, a few more search points are saved but PSNR degradations are obvious in some large motion sequences such as Stefan. Experimental results show that $\mathrm{T}=0.9$ is also an optimum value for the combination SPS(BBGDS, 3SS, T) in sequences of different resolutions. For other SPS combinations, an optimum threshold value $\mathrm{T}$ can be found using the same empirical method demonstrated. Experimental results show that the optimum threshold values T are around 0.85-0.95. It agrees with the statistics in Section III.

\section{B. Comparison of SPS(SDS, 3SS, T) With E3SS}

SPS(SDS, 3SS, T) uses the same small and large motion search patterns as in E3SS, which is in effect a combination 
TABLE V

PERFormance Comparison OF SPS With OTHER FBMAs (CIF SEQUENCES)

\begin{tabular}{|c|c|c|c|c|c|c|c|c|c|c|}
\hline & \multicolumn{2}{|c|}{ Akiyo } & \multicolumn{2}{|r|}{ News } & \multicolumn{2}{|c|}{ Coastguard } & \multicolumn{2}{|c|}{ Foreman } & \multicolumn{2}{|c|}{ Stefan } \\
\hline & $\begin{array}{c}\text { PSNR } \\
(\mathrm{db})\end{array}$ & $\begin{array}{c}\text { \# of search } \\
\text { points }\end{array}$ & $\begin{array}{c}\text { PSNR } \\
(\mathrm{db})\end{array}$ & $\begin{array}{c}\text { \# of search } \\
\text { points }\end{array}$ & $\begin{array}{c}\text { PSNR } \\
(\mathrm{db})\end{array}$ & $\begin{array}{c}\text { \# of search } \\
\text { points }\end{array}$ & $\begin{array}{c}\text { PSNR } \\
(\mathrm{db})\end{array}$ & $\begin{array}{c}\text { \# of search } \\
\text { points }\end{array}$ & $\begin{array}{c}\text { PSNR } \\
(\mathrm{db})\end{array}$ & $\begin{array}{c}\text { \# of search } \\
\text { points }\end{array}$ \\
\hline FS & 43.044 & 983.919 & 36.972 & 983.919 & 30.037 & 983.919 & 32.881 & 983.919 & 25.724 & 983.919 \\
\hline $3 \mathrm{SS}$ & 42.919 & 30.616 & 36.607 & 30.618 & 29.397 & 30.784 & 31.920 & 30.776 & 24.042 & 30.763 \\
\hline E3SS & 43.011 & 12.312 & 36.691 & 12.982 & 29.882 & 17.706 & 32.188 & 19.233 & 24.258 & 21.111 \\
\hline BBGDS & 43.040 & 8.521 & 36.660 & 9.247 & 29.809 & 13.718 & 32.140 & 16.725 & 23.575 & 15.516 \\
\hline DS & 43.025 & 11.286 & 36.694 & 11.898 & 29.879 & 16.541 & 32.096 & 17.925 & 24.033 & 17.608 \\
\hline CDS & 43.011 & 8.700 & 36.677 & 9.523 & 29.879 & 15.682 & 32.014 & 16.686 & 23.952 & 16.958 \\
\hline $\begin{array}{c}\text { SPS } \\
(\mathrm{BBGDS}, 3 \mathrm{SS}, 0.9)\end{array}$ & 43.018 & 5.076 & 36.730 & 6.343 & 29.889 & 13.034 & 32.300 & 15.648 & 24.231 & 17.045 \\
\hline $\begin{array}{c}\text { SPS } \\
(\mathrm{SDS}, 3 \mathrm{SS}, 0.9)\end{array}$ & 43.010 & 5.019 & 36.686 & 6.123 & 29.884 & 11.437 & 32.178 & 13.949 & 24.218 & 16.171 \\
\hline $\begin{array}{c}\text { SPS } \\
\text { (DS, 4SS, 0.9) }\end{array}$ & 43.018 & 5.315 & 36.724 & 6.620 & 29.881 & 14.893 & 32.184 & 16.272 & 24.297 & 17.092 \\
\hline $\begin{array}{c}\text { Speedup of SPS } \\
\text { (SDS, 3SS, 0.9) over } \\
\text { E3SS (\%) }\end{array}$ & \multicolumn{2}{|c|}{$59.24 \%$} & \multicolumn{2}{|c|}{$52.83 \%$} & \multicolumn{2}{|c|}{$35.40 \%$} & \multicolumn{2}{|c|}{$27.47 \%$} & \multicolumn{2}{|c|}{$23.40 \%$} \\
\hline
\end{tabular}

TABLE VI

Performance Comparison BeTWeEn SPS AND UMHEXAGonS

\begin{tabular}{c|c|c|c|c|c|c|c|c|c|c|c|c}
\hline & \multicolumn{3}{|c|}{ Akiyo } & \multicolumn{3}{c|}{ News } & \multicolumn{3}{c}{ Coastguard } & \multicolumn{2}{c}{ Stefan } \\
\hline & $\begin{array}{c}\text { PSNR } \\
(\mathrm{dB})\end{array}$ & $\begin{array}{c}\text { bitrate } \\
(\mathrm{kbits} / \mathrm{s})\end{array}$ & $\begin{array}{c}\text { run time } \\
(\mathrm{sec})\end{array}$ & $\begin{array}{c}\text { PSNR } \\
(\mathrm{dB})\end{array}$ & $\begin{array}{c}\text { bitrate } \\
(\mathrm{kbits} / \mathrm{s})\end{array}$ & $\begin{array}{c}\text { run time } \\
(\mathrm{sec})\end{array}$ & $\begin{array}{c}\text { PSNR } \\
(\mathrm{dB})\end{array}$ & $\begin{array}{c}\text { bitrate } \\
(\mathrm{kbits} / \mathrm{s})\end{array}$ & $\begin{array}{c}\text { run time } \\
(\mathrm{sec})\end{array}$ & $\begin{array}{c}\text { PSNR } \\
(\mathrm{dB})\end{array}$ & $\begin{array}{c}\text { bitrate } \\
(\mathrm{kbits} / \mathrm{s})\end{array}$ & $\begin{array}{c}\text { run time } \\
(\mathrm{sec})\end{array}$ \\
\hline FS & 40.00 & 404.33 & 371.86 & 38.35 & 698.41 & 373.35 & 34.45 & 1896.58 & 373.88 & 35.22 & 2550.76 & 490.68 \\
\hline $\begin{array}{c}\text { UMHexagonS } \\
\text { SPS }\end{array}$ & 39.99 & 404.38 & 10.27 & 38.35 & 697.99 & 11.16 & 34.44 & 1892.34 & 17.43 & 35.21 & 2553.82 & 15.92 \\
\hline $\begin{array}{c}\text { SBGS, 3SS, 0.9) } \\
\text { Speedup over } \\
\text { UMHexagonS }\end{array}$ & 39.97 & 404.72 & 9.17 & 38.34 & 700.25 & 9.37 & 34.44 & 1893.06 & 10.15 & 35.20 & 2570.30 & 10.21 \\
\hline
\end{tabular}

of SDS and 3SS. The only difference is the motion content classifier. SPS(SDS, 3SS) uses the proposed EDR, while E3SS uses initial search pattern as its geometric motion content classifier. The performances of SPS(SDS, 3SS, 0.9) and E3SS are shown in Table V. The last row of the table shows the speedup of SPS(SDS, 3SS, 0.9) over E3SS. It can be seen that SPS(SDS, 3SS, 0.9) is $23.40-59.24 \%$ faster than E3SS. The matching qualities of both algorithms are similar.

\section{Comparison With Other Motion Estimation Algorithms}

Table V compares the performance of SPS(BBGDS, 3SS, 0.9), SPS(SDS, 3SS, 0.9), and SPS(DS, 4SS, 0.9) with FS, 3SS, E3SS, BBGDS, DS, and CDS using the CIF sequences. Among the three SPS combinations, SPS(BBGDS, 3SS, 0.9) performs best. Compared with other FBMAs, SPS(BBGDS, 3SS, 0.9) has the highest PSNR for News, Coastguard, and Foreman. It uses the fewest search points for Akiyo, News, Coastguard, and Foreman. Experiments were also conducted using the QCIF and CCIR601 sequences. Results also show that SPS is much faster than other algorithms.

\section{Implementation in H.264 Reference Software}

SPS(BBGDS, 3SS, 0.9) is implemented in the H.264 reference software JM9.6 [16] to compare with UMHexagonS [17]. UMHexagonS combines many techniques, e.g., motion vector prediction and early termination, from different ME research fields. The experimental setup is IPPPIPPP frame structure, Hadamard transform, and one reference frame. Table VI compares SPS(BBGDS, 3SS, 0.9) with UMHexagonS. The average PSNR and bit rate (kbits/s) are used for video quality evaluation. The computational complexity is measured by the ME encoding time. As shown, SPS is faster than UMHexagonS with similar bit-rate performance.

\section{CONCLUSION}

A new motion content classifier using EDR is proposed in this paper. Based on this, the SPS algorithm is proposed. Compared to other recently proposed adaptive algorithms, SPS algorithm has negligible algorithm decision overhead. Experimental results show that the SPS algorithm is very robust. 


\section{REFERENCES}

[1] T. Wiegand, G. J. Sullivan, G. Bjontegaard, and A. Luthra, "Overview of the H.264/AVC video coding standard," IEEE Trans. Circuits Syst. Video Technol., vol. 13, no. 7, pp. 560-576, Jul. 2003.

[2] J. R. Jain and A. K. Jain, "Displacement measurement and its application in interframe image coding," IEEE Trans. Commun., vol. 29, no. 12, pp. 1799-1808, Dec. 1981.

[3] T. Koga, K. Iinuma, A. Hirano, Y. Iijima, and T. Ishiguro, "Motion compensated interframe coding for video conferencing," in Proc. Nat. Telecommun. Conf., New Orleans, LA, Nov.-Dec. 1981, pp. G.5.3.1G.5.3.5.

[4] C. H. Cheung and L. M. Po, "A novel cross-diamond search algorithm for fast block motion estimation," IEEE Trans. Circuits Syst. Video Technol., vol. 12, no. 12, pp. 1168-177, Dec. 2002.

[5] R. Li, B. Zeng, and M. L. Lio, "A new three-step search algorithm for block motion estimation," IEEE Trans. Circuits Syst. Video Technol., vol. 4, no. 4, pp. 438-442, Aug. 1994.

[6] L. M. Po and W. C. Ma, "A novel four-step search algorithm for fast block motion estimation," IEEE Trans. Circuits Syst. Video Technol., vol. 6, no. 3, pp. 313-317, Jun. 1996.

[7] L. K. Liu and E. Feig, "A block-based gradient descent search algorithm for block motion estimation in video coding," IEEE Trans. Circuits Syst. Video Technol., vol. 6, no. 4, pp. 419-422, Aug. 1996.

[8] J. Y. Tham, S. Ranganath, M. Ranganath, and A. A. Kassim, "A novel unrestricted center-biased diamond search algorithm for block motion estimation," IEEE Trans. Circuits Syst. Video Technol., vol. 8, no. 4, pp. 369-377, Aug. 1998.
[9] J.-F. Yang, S.-C. Chang, and C.-Y. Chen, "Computation reduction for motion search in low rate video coders," IEEE Trans. Circuits Syst. Video Technol., vol. 12, no. 12, pp. 948-951, Oct. 2002.

[10] L. Yang, K. Yu, J. Li, and S. Li, "An effective variable block-size early termination algorithm for H.264 video coding," IEEE Trans. Circuits Syst. Video Technol., vol. 15, no. 6, pp. 784-788, Jun. 2005.

[11] X. Jing and L. P. Chau, "An efficient three-step search algorithm for block motion estimation," IEEE Trans. Multimedia, vol. 6, no. 3, pp. 435438, Jun. 2004.

[12] C. S. Yu and S. C. Tai, "Adaptive Double-Layered Initial Search Pattern for Fast Motion Estimation," IEE Trans. Multimedia, vol. 8, no. 6 , pp. 1109-1116, Dec. 2006.

[13] Y. Nie and K. K. Ma, "Adaptive irregular pattern search with matching prejudgement for fast block-matching motion estimation," IEEE Trans. Circuits Syst. Video Technol., vol. 15, no. 6, pp. 789-794, Jun. 2005.

[14] J. H. Lim and H. W. Choi, "Adaptive motion estimation algorithm using spatial and temporal correlation," in Proc. IEEE Pacific Rim Conf. Commun., Comput. Signal Process. 2001, vol. 2. Victoria, BC, Aug. 2001, pp. 473-476.

[15] S.-Y. Huang, C.-Y. Cho, and J.-S. Wang, "Adaptive fast block-matching algorithm by switching search patterns for sequences with wide-range motion content," IEEE Trans. Circuits Syst. Video Technol., vol. 15, no. 11, pp. 1373-1384, Nov. 2005.

[16] Joint Video Team (JVT) reference software version 9.6 [Online]. Available: http://iphome.hhi.de/suehring/tml/download/old_jm/

[17] Z. B. Chen, P. Zhou, and Y. He, "Fast Motion Estimation for JVT," JVTG016.doc, Joint Video Team (JVT) of ISO/IEC MPEG \& ITU-T VCEG, 7th Meeting: Pattaya II, Thailand, Mar. 7-14, 2003. 